\title{
Elective frozen embryo transfer (freeze-all): there seems to be no harm to transfer in the next immediate menstrual cycle
}

\author{
Matheus Roque ${ }^{1}$, Sandro C. Esteves ${ }^{2,3} \wedge$ \\ ${ }^{1}$ Mater Prime - Reproductive Medicine, São Paulo, Brazil; ${ }^{2}$ Androfert, Andrology and Human Reproduction Clinic, Campinas, SP, Brazil; ${ }^{3}$ Faculty of \\ Health, Aarhus University, Aarhus, Denmark \\ Correspondence to: Sandro C. Esteves. Av. Dr. Heitor Penteado, 1464, 13075-460 Campinas, SP, Brazil. Email: s.esteves@androfert.com.br. \\ Provenance and Peer Review: This article was commissioned by the editorial office, Annals of Translational Medicine. The article did not undergo \\ external peer review. \\ Comment on: Huang J, Lu X, Xie Q, et al. Timing of frozen-thawed embryo transfer after controlled ovarian stimulation in a non-elective freeze-all \\ policy. Ann Transl Med 2019;7:752.
}

Submitted Feb 29, 2020. Accepted for publication Apr 21, 2020.

doi: $10.21037 /$ atm-20-2070

View this article at: http://dx.doi.org/10.21037/atm-20-2070

The development of cryopreservation techniques is among the most significant advances in assisted reproductive technology (ART). Novel vitrification protocols provide higher post-warming embryo survival than conventional cryopreservation techniques, and frozen embryo transfer (FET) yields comparable -or higher- pregnancy outcomes than fresh ET $(1,2)$. As a result, new metrics emerged, like the cumulative live birth rate (CLBR) per cycle, which refers to live births resulting from one ART cycle, including all cycles in which fresh and/or frozen embryos are transferred (3). The CLBR metric is one of the pillars of the novel POSEIDON criteria for low prognosis women undergoing ART, which has gained increased interest among the reproductive medicine community $(3,4)$. More importantly, vitrification is the mainstay of elective FET (eFET) strategy, so-called 'freeze-all', in which all viable embryos resulting from the IVF process are electively cryopreserved $(2,5)$.

Elective FET has transformed the way we perform ART nowadays. Clinicians can now easily avoid its most feared complication, that is, ovarian hyperstimulation syndrome (OHSS), thus making the OHSS-free Clinic a reality. In the United States, the number of freeze-all cycles increased 33 times in the last ten years, from 2,020 in 2007 to 65,840 in 2016 (1). In that country, $25 \%$ of all cycles in 2016 were eFET, whereas the corresponding figure for Europe was $15 \%$. It is therefore clear that several fertility clinics have adopted a liberal approach towards eFET, increasing its overall use.

The most accorded indication for eFET is the risk of OHSS. In this scenario, ovarian stimulation (OS) carried out with the use of a GnRH antagonist protocol and the $\mathrm{GnRH}$ agonist for final oocyte maturation, followed by eFET, decreases OHSS to virtually $0 \%$ whereas securing similar pregnancy rates as compared to fresh ET (6).

However, concerns of possible deleterious effects of OS on the endometrium owed to supra-physiological steroid levels have led researchers to investigate the effect of eFET on a broad population of women undergoing ART. Patients subjected to OS might present histological endometrial advancement, changes in endometrium gene expression, or progesterone elevation in the late follicular phase, all of which could adversely affect embryo implantation albeit the evidence is not unequivocal (7-10).

Yet, despite the significant universal shift towards eFET, the evidence for its generalized outcome benefit and safety remains sparse. The first studies comparing eFET and fresh ET in normal responders suggested the superiority of eFET with regards to clinical pregnancy (CPR) and ongoing pregnancy rates (OPR) (11). Subsequent randomized controlled trials (RCT) have reported mixed results on reproductive outcomes, in particular, among women with polycystic ovary syndrome (PCOS) (12), normo-ovulatory women $(13,14)$, non-PCOS women $(15)$, and patients undergoing preimplantation genetic testing for aneuploidy

^ ORCID: 0000-0002-1313-9680 
(PGT-A) (16). In a 2019 meta-analysis including 5,379 patients randomized to freeze-all or fresh ET cycle, we found an overall $7 \%$ increase in live birth rate (LBR) favoring the eFET strategy ( $R R=1.07 ; \mathrm{P}=0.02)$ (5). However, this increase was not confirmed when CLBR was the endpoint, and our subgroup analyses indicated that eFET was only advantageous for hyper-responders $(\mathrm{RR}=1.15 ; \mathrm{P}=0.005)$ and PGT-A patients.

Thus, based on the data accumulated over the last seven years, it seems that the indiscriminate use of eFET does not confer additional benefit over fresh ET $(2,5,17)$. Notwithstanding these observations, no RCTs exist concerning eFET in poor responders. In this scenario, data from observational studies failed to demonstrate an association between eFET and increase pregnancy outcomes (18). Yet, eFET might be beneficial for poor prognosis patients pooling embryos as a way to increase the number of embryos available for transfer or PGT-A. As for eFET in patients with elevated progesterone levels on the day of trigger, data are also scarce, although a sub-analysis of Vuong et al. RCT mentioned above (15) seem to indicate a positive effect of eFET on LBRs (19). Other possible indications for eFET include slow embryo development, which might affect the synchrony between embryo and endometrium implantation window in fresh ET, presence of endometrial, tubal, or uterine factors, endometriosis, adenomyosis, and oocyte or embryo pooling in patients with low prognosis. At present, no RCT exists to support or refute the use of eFET in the conditions mentioned above, thus deserving further investigation.

A critical issue concerning eFET is the safety of mother and baby. The literature seems to indicate that FET cycles are associated with an increased risk of pregnancy complications, including hypertension, preeclampsia, and macrosomia. By contrast, fresh ET has been associated with an increased risk of preterm birth, low birth weight, and small for gestational age. We have speculated that the type of endometrial priming rather than the cryopreservation per se is the cause of the adverse obstetric and perinatal outcomes observed in eFET pregnancies (20). It is wellknown that the corpus luteum (CL) secretes vasoactive hormones that play a fundamental role during the early pregnancy maternal circulation. Unlike in the natural cycles, the CL is absent in artificial cycles due to the endometrial preparation with estrogens and progesterone.

Interestingly, some data suggest an increased risk of preeclampsia in pregnancies from FET in artificial cycles (21).
Moreover, data from a large population-based retrospective registry study, which compared outcomes of singletons after FET versus fresh ET, and with natural conception singletons, showed an increased risk of hypertensive disorders and macrosomia in FET cycles (22). The observed increased risk was related to the absence of the CL in FET transfers. Thus, the type of endometrial priming is a variable to be controlled for in FET studies looking at gestational and neonatal complications.

Another critical issue concerns the optimal time to perform embryo transfers after freeze-all. Delaying ET too much might increase the time-to-pregnancy (TTP) and create further patient distress. The first studies on the matter concerned showed that pregnancy outcomes were not significantly different in FET cycles performed in the next menstrual cycle after 'freeze-all' or later $(23,24)$.

In a recent paper published in the Annals of Translational Medicine, Huang et al. shed light on the latter, by retrospectively evaluating a large cohort of 2,998 patients subjected eFET (25). In their study, eFETs carried out within the first menstrual cycle following OS and oocyte retrieval were associated with a higher chance to achieve a live birth than delaying FET to subsequent cycles. The authors' results add to the literature, albeit contrasting with previous observations. The reasons related to the observed increased likelihood of achieving a live birth after immediate eFET remain unknown, thus deserving further research. Moreover, the confirmation of Huang et al.'s findings by a well-conducted RCT is needed. In the meanwhile, the study of Huang and colleagues can help doctors reassure their patients undergoing eFET that there is no need to delay ET.

In conclusion, the indiscriminate use of eFET has shortcomings. The best course of action is to be judicious and individualize eFET. Currently, the best candidates are patients with risk of OHSS, hyper-responders/ PCOS patients, and patients whose embryos will undergo trophectoderm biopsy. The ideal regimen for endometrial preparation in eFET cycles is yet to be determined, and its impact on gestational and neonatal outcomes remains an open question. While waiting for further RCTs, it seems sound to avoid delaying FET whenever possible as ETs performed in the next cycle (after freeze-all) might not only reduce the TTP but also increase pregnancy prospects.

\section{Acknowledgments}

Funding: None. 


\section{Footnote}

Conflicts of Interest: Both authors have completed the ICMJE uniform disclosure form (available at http://dx.doi. org/10.21037/atm-20-2070). SCE and MR report grants and personal fees from Merck, outside the submitted work.

Ethical Statement: The authors are accountable for all aspects of the work in ensuring that questions related to the accuracy or integrity of any part of the work are appropriately investigated and resolved.

Open Access Statement: This is an Open Access article distributed in accordance with the Creative Commons Attribution-NonCommercial-NoDerivs 4.0 International License (CC BY-NC-ND 4.0), which permits the noncommercial replication and distribution of the article with the strict proviso that no changes or edits are made and the original work is properly cited (including links to both the formal publication through the relevant DOI and the license). See: https://creativecommons.org/licenses/by-nc-nd/4.0/.

\section{References}

1. Nagy ZP, Shapiro D, Chang CC. Vitrification of the human embryo: a more efficient and safer in vitro fertilization treatment. Fertil Steril 2020;113:241-7.

2. Sciorio R, Esteves SC. Clinical utility of freeze-all approach in ART treatment: A mini-review. Cryobiology 2020;92:9-14.

3. Esteves SC, Alviggi C, Humaidan P, et al. The POSEIDON Criteria and Its Measure of Success Through the Eyes of Clinicians and Embryologists. Front Endocrinol (Lausanne) 2019;10:814.

4. Esteves SC, Yarali H, Ubaldi FM, et al. Validation of ART Calculator for Predicting the Number of Metaphase II Oocytes Required for Obtaining at Least One Euploid Blastocyst for Transfer in Couples Undergoing in vitro Fertilization/Intracytoplasmic Sperm Injection. Front Endocrinol (Lausanne) 2020;10:917.

5. Roque M, Haahr T, Geber S, et al. Fresh versus elective frozen embryo transfer in IVF/ICSI cycles: a systematic review and meta-analysis of reproductive outcomes. Hum Reprod Update 2019;25:2-14.

6. Devroey P, Polyzos NP, Blockeel C. An OHSS-free clinic by segmentation of IVF treatment. Hum Reprod 2011;26:2593-7.

7. Ubaldi F, Bourgain C, Tournaye H, et al. Endometrial evaluation by aspiration biopsy on the day of oocyte retrieval in the embryo transfer cycles in patients with serum progesterone rise during follicular phase. Fertil Steril 1997;67:521-6.

8. Horcajadas JA, Riesewijk A, Polman J, et al. Effect of controlled ovarian hyperstimulation in IVF on endometrial gene expression profiles. Mol Hum Reprod 2005;11:195-205.

9. Labarta E, Martínez-Conejero JA, Alamá P, et al. Endometrial receptivity is affected in women with high circulating progesterone levels at the end of the follicular phase: a functional genomics analysis. Hum Reprod 2011;26:1813-25.

10. Esteves SC, Khastgir G, Shah J, et al. Association Between Progesterone Elevation on the Day of Human Chronic Gonadotropin Trigger and Pregnancy Outcomes After Fresh Embryo Transfer in In Vitro Fertilization/ Intracytoplasmic Sperm Injection Cycles. Front Endocrinol (Lausanne) 2018;9:201.

11. Roque M, Lattes K, Serra S, et al. Fresh embryo transfer in in vitro fertilization cycles: a systematic review and meta-analysis. Fertil Steril 2013;99:156-62.

12. Chen ZJ, Shi Y, Sun Y, et al. Fresh versus Frozen Embryos for Infertility in the Polycystic Ovary Syndrome. N Engl J Med 2016;375:523-33.

13. Shi Y, Sun $Y$, Hao C, et al. Transfer of Fresh versus Frozen Embryos in Ovulatory Women. N Engl J Med 2018;378:126-36.

14. Wei D, Liu JY, Sun Y, et al. Frozen versus fresh single blastocyst transfer in ovulatory women: a multicentre, randomised controlled trial. Lancet 2019;393:1310-8.

15. Vuong LN, Dang VQ, Ho TM, et al. IVF Transfer of Fresh or Frozen Embryos in Women without Polycystic Ovaries. N Engl J Med 2018;378:137-47.

16. Coates A, Kung A, Mounts E, et al. Optimal euploid embryo transfer strategy, fresh versus frozen, after preimplantation genetic screening with next generation sequencing: a randomized controlled trial. Fertil Steril 2017;107:723-730.e3.

17. Roque M, Nuto Nóbrega B, Valle M, et al. Freeze-all strategy in IVF/ICSI cycles: an update on clinical utility. Panminerva Med 2019;61:52-7.

18. Roque M, Valle M, Sampaio $M$, et al. Does freeze-all policy affect IVF outcome in poor ovarian responders? Ultrasound Obstet Gynecol 2018;52:530-4.

19. Vuong LN, Pham TD, Dang VQ, et al. Live birth rates with a freeze-only strategy versus fresh embryo transfer: secondary analysis of a randomized clinical trial. Reprod 
Biomed Online 2019;38:387-96.

20. Roque M, Bedoschi G, Cecchino GN, et al. Fresh versus frozen blastocyst transfer. Lancet 2019;394:1227-8.

21. von Versen-Höynck F, Schaub AM, Chi YY, et al. Increased Preeclampsia Risk and Reduced Aortic Compliance With In Vitro Fertilization Cycles in the Absence of a Corpus Luteum. Hypertension 2019;73:640-9.

22. Ginström Ernstad E, Wennerholm UB, Khatibi A, et al. Neonatal and maternal outcome after frozen embryo transfer: increased risks in programmed cycles. Am J Obstet Gynecol 2019;221:126.e1-126.e18.

Cite this article as: Roque M, Esteves SC. Elective frozen embryo transfer (freeze-all): there seems to be no harm to transfer in the next immediate menstrual cycle. Ann Transl Med 2020;8(15):913. doi: 10.21037/atm-20-2070
23. Lattes K, Checa MA, Vassena R, et al. There is no evidence that the time from egg retrieval to embryo transfer affects live birth rates in a freeze-all strategy. Hum Reprod 2017;32:368-74.

24. Santos-Ribeiro S, Polyzos NP, Lan VT, et al. The effect of an immediate frozen embryo transfer following a freeze-all protocol: a retrospective analysis from two centres. Hum Reprod 2016;31:2541-8.

25. Huang J, Lu X, Xie Q, et al. Timing of frozen-thawed embryo transfer after controlled ovarian stimulation in a non-elective freeze-all policy. Ann Transl Med 2019;7:752. 